\title{
HEAT BALLS AND FULKS MEASURES
}

\author{
HEINZ BAUER
}

\section{Introduction}

Euclidean spheres in $\boldsymbol{R}^{p}$ play an important role in classical potential theory, the theory of the Laplace equation. They enter into the picture as soon as one treats the Poisson formula which is the key to the definition of superharmonic functions. For the Laplace equation, the fundamental solution $N$ with pole at the origin is in every dimension $p \geqq 1$ a function of the euclidean norm of the point $x \in \boldsymbol{R}^{p}$. Hence spheres with center at the origin can be considered as the level surfaces of $N$.

The potential theory of the heat equation

$$
\Delta h-\frac{\partial h}{\partial t}=0
$$

in $\boldsymbol{R}^{p+1}=\boldsymbol{R}^{p} \times \boldsymbol{R}$ is now well developed. In particular, there are formulas analogous to the Poisson formula. However, there is one crucial contrast to the Laplace case: In principle, $(p+1)$-dimensional balls could be used since all boundary points turn out to be regular. However, the proof of this fact demands already quite a bit of potential-theoretic machinery. So, for the definition of supercaloric functions - the analogue of superharmonic functions - , one usually uses either rectangular domains parallel to the coordinate axes where the inconvenience of having irregular boundary points is not serious or truncated rotational cones having a base parallel to the hyperplane $\boldsymbol{R}^{p} \times\{0\}$ and a vertex situated in positive time direction above this base. In both approaches the formula analogous to the Poisson formula is not easy to obtain since integral equations have to be solved. In any case, the final Poisson-type formula contains functions which cannot be written down explicitly.

In view of these difficulties it seems natural to proceed by analogy with the case of the Laplace equation: One considers

$$
W(x, t)= \begin{cases}(-4 \pi t)^{-p / 2} e^{\|x\| 2 / 4 t} & \text { when } t<0 \\ 0 & \text { when } t \geqq 0\end{cases}
$$

- which is the fundamental solution of the adjoint heat equation with pole at the origin $\mathbf{0}$ - and one studies the level surfaces

$$
W(x, t)=d
$$


where $d$ is a positive constant. This idea, due to Fulks [10], will be fundamental for us. In [10] Fulks considers the smooth convex surface $B_{d}$ defined by (*) with inclusion of the limit point $\mathbf{0}$. It is the boundary of the relatively compact domain $\Omega_{d}$ in $\boldsymbol{R}^{p+1}$ defined by

$$
W(x, t)>d .
$$

We shall call $\Omega_{d}$ a heat ball and $B_{d}$ the corresponding heat sphere. In contrast to the case of the Laplace equation, the pole $\mathbf{0}$ of $W$ lies on the heat sphere $B_{d}$. The heat ball $\Omega_{d}$ is an egg-shaped domain which has $\boldsymbol{R}^{p} \times\{0\}$ as tangent hyperplane through $\mathbf{0}$ and which lies in the half-space of all points $(x, t)$ with negative time coordinate $t$. In [10] Fulks defines explicitly a continuous function $Q_{d} \geqq 0$ on $B_{d}$ which is strictly positive with the exception of one point. The measure $\mu_{d}$ which has $Q_{d}$ as density with respect to the surface measure of $B_{d}$ is a probability measure. This Fulks measure $\mu_{d}$ is connected with the solutions of the heat equation in a similar way as the normalized surface measure $\sigma_{r}$ of the sphere $\|x\|=r$ in $\boldsymbol{R}^{p}$ is connected with harmonic functions, however with one important difference: For the Laplace equation the usual mean value property says that integration of a harmonic function with respect to $\sigma_{r}$ yields the value of the function at the origin, hence at the center of the euclidean ball $\|x\|<r$. For solutions of the heat equation integration with respect to $\mu_{d}$ yields the value of the solution again at the origin $\mathbf{0}$ which is now a point of the heat sphere. According to [10], solutions of the heat equation can be characterized by mean value formulas where one integrates with respect to measures which are obtained from $\mu_{d}$ by translations. Also these translated measures will be called Fulks measures. Furthermore, Watson [18], [19] uses these measures for the definition and study of supertemperatures which correspond to superharmonic functions for the Laplace equation. However, no attempt seems to have been made to identify these supertemperatures with the supercaloric (or superparabolic) functions which are defined by means of the above mentioned rectangular domains or truncated cones, and hence in agreement with the general definition of superharmonic functions in the theory of harmonic spaces.

By making essential use of the harmonic space theory for the heat equation, we intend to show as the main result of this paper that every Fulks measure $\mu_{d}$ allows a simple potential-theoretic interpretation: $\mu_{d}$ is obtained from the unit mass at $\mathbf{0}$ by sweeping this mass out to the complement of $\Omega_{d}$, i.e.,

$$
\mu_{d}=\varepsilon_{0}^{\mathbf{c} \Omega_{d}} .
$$

In the proof we use a result of Boboc and Cornea [6] about cluster values of harmonic measures, a result which has been improved recently by Lukeš and Malý [16]. Our main result has several consequences which are interesting in themselves: The result implies that the origin $\mathbf{0}$ is an irregular boundary point of the heat ball $\Omega_{d}$ and that supertemperatures coincide with supercaloric functions. Furthermore, our result leads to a probabilistic interpretation of the Fulks measures: $\mu_{d}$ is the distribution 
of the first hit of the heat sphere $B_{d}$ (at times $t>0$ ) by the Brownian heat transfer starting at $\mathbf{0}$, where the Brownian heat transfer in $\boldsymbol{R}^{p+1}$ is the well-known space-time modification of $p$-dimensional Brownian motion. So, altogether, the measures $\mu_{d}$ find a very satisfactory and natural potential-theoretic characterization.

In order to make our presentation as smoothly accessible as possible, the paper is organized as follows: After the introduction of the Fulks measures we state our Main Theorem, namely the characterization of $\mu_{d}$. Then we immediately give applications. Finally, the proof of the Main Theorem is presented in the third and last paragraph of this paper.

In our presentation the harmonic space associated to the heat equation and the corresponding potential theory is used constantly and without further explanations. Standard references are [1], [3] and [7].

The main results of this paper have been announced in C.R. Acad. Sci. Paris, Sér. A.

\section{Definition of the Fulks measures}

We shall be concerned with the heat equation

$$
\Delta h-\frac{\partial h}{\partial t}=0
$$

in $\boldsymbol{R}^{p+1}=\boldsymbol{R}^{p} \times \boldsymbol{R}$ for $p=1,2, \ldots$ The points of $\boldsymbol{R}^{p+1}$ will be denoted correspondingly by $(x, t)=\left(x_{1}, \ldots, x_{p}, t\right)$ where $x=\left(x_{1}, \ldots, x_{p}\right) \in \boldsymbol{R}^{p}$ and $t \in \boldsymbol{R}$. In particular, the Laplace operator refers to the first $p$ coordinates:

$$
\Delta=\sum_{i=1}^{p} \frac{\partial^{2}}{\partial x_{i}^{2}}
$$

The euclidean norm of a point $x=\left(x_{1}, \ldots, x_{p}\right) \in \boldsymbol{R}^{p}$ will be denoted by

$$
\|x\|=\left(x_{1}^{2}+\ldots+x_{p}^{2}\right)^{1 / 2} .
$$

Solutions of (1.1), more precisely functions $h: U \rightarrow \boldsymbol{R}$ defined on an open subset $U$ of $\boldsymbol{R}^{p+1}$ which are of class $C^{2}$ with respect to the space variable $x$ and of class $C^{1}$ with respect to the time variable $t$ and which satisfy (1.1), will be called caloric functions or temperatures.

We denote by $\tilde{W}$ the fundamental solution of (1.1) with pole at the origin $\mathbf{0}=$ $(0,0)$, i.e.,

$$
\tilde{W}(x, t)=\left\{\begin{array}{lll}
\left(\frac{1}{4 \pi t}\right)^{p / 2} e^{-\|x\| 2 / 4 t} & \text { when } & t>0 \\
0 & \text { when } t \leqq 0 .
\end{array}\right.
$$

Reflection at $\mathbf{0}$ yields the function $W$ from the introduction:

$$
W(x, t)=\tilde{W}(-x,-t) .
$$


By translation we obtain for every point $z_{0}=\left(x_{0}, t_{0}\right) \in \boldsymbol{R}^{p+1}$ the function

$$
W_{z_{0}}(x, t)=\tilde{W}\left(x_{0}-x, t_{0}-t\right)
$$

which is the fundamental solution of the adjoint heat equation

$$
\Delta h+\frac{\partial h}{\partial t}=0
$$

with pole at $z_{0}$. In particular, $W=W_{0}$.

Given $z_{0} \in \boldsymbol{R}^{p+1}$ and a real number $c>0$ we denote by $\Omega\left(z_{0}, c\right)$ the set of all points $(x, t) \in \boldsymbol{R}^{p+1}$ satisfying

$$
W_{z_{0}}(x, t)>\left(\frac{1}{4 \pi c}\right)^{p / 2}
$$

This set $\Omega\left(z_{0}, c\right)$ is a relatively compact domain, in fact a convex body. Its boundary

$$
B\left(z_{0}, c\right)=\partial \Omega\left(z_{0}, c\right)
$$

is a smooth surface given by the equation

$$
W_{z_{0}}(x, t)=\left(\frac{1}{4 \pi c}\right)^{p / 2}
$$

with inclusion of the point $z_{0}$. More precisely, let us define for $c>0$ the (continuous) function $F_{c}:[0, c] \rightarrow \boldsymbol{R}$ by

$$
F_{c}(t)=\left\{\begin{array}{lll}
{\left[-2 p t \log \frac{t}{c}\right]^{1 / 2}} & \text { when } \quad 0<t \leqq c \\
0 & \text { when } t=0
\end{array}\right.
$$

then the surface $B\left(z_{0}, c\right)$ is given by the equation

$$
\left\|x-x_{0}\right\|=F_{c}\left(t_{0}-t\right) \text { with } 0 \leqq t_{0}-t \leqq c .
$$

From this it can be concluded that $B\left(z_{0}, c\right)$ is a ( $p$-dimensional) $C^{2}$-manifold and that $B\left(z_{0}, c\right) \backslash\left\{z_{0}\right\}$ is even a $C^{\infty}$-manifold $\left.{ }^{1}\right)$. The domains $\Omega\left(z_{0}, c\right)$ will be called heat balls in $\boldsymbol{R}^{p+1}$; correspondingly, the manifolds $B\left(z_{0}, c\right)$ will be called heat spheres.

1) Denote by $\Psi_{0}$ the inverse of the strictly decreasing function $\Phi_{0}:\left[0, e^{-1}[\rightarrow] e^{-1 / e}, 1\right]$ where $\Phi_{0}(\tau)=\tau^{\tau}$ for $\tau>0$ and $=1$ for $\tau=0$, and denote by $\Psi_{1}$ the inverse of the strictly increasing function $\left.\Phi_{1}:\right] e^{-1},+\infty[\rightarrow] e^{-1 / e},+\infty\left[\right.$ where $\Phi_{1}(\tau)=\tau^{\tau}$. Then one obtains two charts for neighborhoods of the points $z_{0}$ and $z_{1}=\left(x_{0}, t_{0}-c\right)$, respectively. In fact, it is easy to see that the equations

$$
t=t_{0}-c \Psi_{0}\left(e^{-\frac{\left\|x-x_{0}\right\|^{2}}{2 p c}}\right)
$$

and

$$
t=t_{0}-c \Psi_{1}\left(e^{-\frac{\left\|x-x_{0}\right\|^{2}}{2 p c}}\right)
$$


In what follows $\sigma=\sigma_{B\left(z_{0}, c\right)}$ will denote the surface measure of the heat sphere $B\left(z_{0}, c\right)$. We define a function $Q>0$ on $\left(\boldsymbol{R}^{p} \times\right] 0,+\infty[) \cup\{\mathbf{0}\}$ by means of

$$
Q(x, t)=\left\{\begin{array}{lll}
\|x\|^{2}\left[4\|x\|^{2} t^{2}+\left(\|x\|^{2}-2 p t\right)^{2}\right]^{-1 / 2} & \text { when } t>0 \\
1 & \text { when } \quad(x, t)=\mathbf{0} .
\end{array}\right.
$$

Furthermore, for $z_{0}=\left(x_{0}, t_{0}\right) \in \boldsymbol{R}^{p+1}$ we define a function

$$
q_{z_{0}}(x, t)=Q\left(x_{0}-x, t_{0}-t\right)
$$

on $\left(\boldsymbol{R}^{p} \times\right]-\infty, t_{0}[) \cup\left\{z_{0}\right\}$. Its restriction to $B\left(z_{0}, c\right)$

$$
q_{z_{0}, c}=q_{z_{0}} \mid B\left(z_{0}, c\right)
$$

turns out to be a continuous function which is strictly positive on $B\left(z_{0}, c\right)$ with the only exception of the point $z_{1}=\left(x_{0}, t_{0}-c\right)$ where $q_{z_{0}}\left(z_{1}\right)=0$. By taking $q_{z_{0}, c}$ as a density with respect to $\sigma$ we finally obtain the main object of our investigation.

1.1 Definition. The positive Radon measure

$$
\mu_{z_{0}, c}=\left(\frac{1}{4 \pi c}\right)^{p / 2} q_{z_{0}, c} \sigma_{B\left(z_{0}, c\right)}
$$

on $B\left(z_{0}, c\right)$ is called the Fulks measure for the heat ball $\Omega\left(z_{0}, c\right)$.

According to Fulks [10], $\mu_{z_{0}, c}$ is a probability measure. In fact, this follows from the following fundamental result of [10]:

(F) Every caloric function $h$ defined in a domain $D$ of $\boldsymbol{R}^{p+1}$ satisfies the following mean value property:

$$
\int h d \mu_{z_{0}, c}=h\left(z_{0}\right)
$$

whenever $z_{0} \in D$ and $\bar{\Omega}\left(z_{0}, c\right) \subset D$.

in both cases with

describe the neighborhoods

$$
\left\|x-x_{0}\right\|<\sqrt{2 p \frac{c}{e}}
$$

and

$$
U_{0}=B\left(z_{0}, c\right) \cap\left\{(x, t): t>t_{0}-\frac{c}{e}\right\}
$$

$$
U_{1}=B\left(z_{0}, c\right) \cap\left\{(x, t): t<t_{0}-\frac{c}{e}\right\}
$$

of $z_{0}$ and $z_{1}$, respectively. The right-hand side of (a) is a $C^{2}$-function, the right-hand side of (b) is a $C^{\infty}$-function of $x$. The value $t=t_{0}-c / e$ yields the "equator" of $B\left(z_{0}, c\right)$ which corresponds to the maximum of $F_{c}$ :

$$
F_{c}\left(\frac{c}{e}\right)=\sqrt{2 p \frac{c}{e}} .
$$


In [10] Fulks also shows the converse: A continuous function $h: D \rightarrow \boldsymbol{R}$ on a domain $D$ of $\boldsymbol{R}^{p+1}$ with the above mean value property is caloric. However, (F) as well as its converse will not be needed in the sequel. They will follow from our Main Result and its Addendum (see Corollary 2.3 and 2.6, Remark 2). Let us also remark that the Fulks measures have been rediscovered by Kuptsov [15] in an analytical presentation which, however, is less convenient for our purposes.

\section{The main result and its consequences}

We shall now give a potential-theoretic characterization of the Fulks measure $\mu_{z_{0}, c}$ which will be considered as a Radon measure on $\boldsymbol{R}^{p+1}$ concentrated on $B\left(z_{0}, c\right)$. Potential-theoretic notions for the heat equation should always be understood in the sense of harmonic spaces. More precisely, $\boldsymbol{R}^{p+1}$ is considered as the harmonic space with respect to the harmonic sheaf given by the caloric functions. It is a wellknown fact [1] that this space is strongly harmonic or $\mathfrak{P}$-harmonic in the terminology of Constantinescu and Cornea [7].

We shall start with a simple observation:

2.1 Proposition. All points $z \neq z_{0}$ of the heat sphere $B\left(z_{0}, c\right)$ are regular boundary points of the heat ball $\Omega\left(z_{0}, c\right)$ (for arbitrary $z_{0} \in \boldsymbol{R}^{p+1}$ and $c>0$ ).

Proof. This can be seen in many ways. A simple procedure is the following: The boundary point $z_{1}=\left(x_{0}, t_{0}-c\right)$ with the same space coordinate as $z_{0}$ is regular since, obviously,

$$
v(x, t)=\left\|x-x_{0}\right\|^{2}+2 p\left(t-t_{0}+c\right)
$$

is a caloric barrier for $z_{1}$. For all remaining boundary points $z=(\xi, \tau)$ in $B\left(z_{0}, c\right) \backslash\left\{z_{0}, z_{1}\right\}$ there exists a closed euclidean $(p+1)$-dimensional ball $K$ such that $K \cap \bar{\Omega}\left(z_{0}, c\right)=\{z\}$ and such that $\xi$ is not the space coordinate $\bar{x}$ of the center $(\bar{x}, \bar{t})$ of $K$. It then follows from Friedman [9], p. 69, property (b), that $z$ is regular. In fact for $\alpha>0$ sufficiently large,

where

$$
v(x, t)=R(z)^{-\alpha}-R(x, 1)^{-\alpha},
$$

$$
R(x, t)=\|x-\bar{x}\|^{2}+(t-\bar{\imath})^{2},
$$

is a barrier for $z$ (see also Effros and Kazdan [8], Lemma 2) ${ }^{2}$ ).

Our main result is now the following:

2.2 Main Theorem. For all $z_{0} \in \boldsymbol{R}^{p+1}$ and $c>0$, the Fulks measure $\mu_{z_{0}, c}$ is the unit mass at $z_{0}$ swept out to the complement of $\Omega=\Omega\left(z_{0}, c\right)$, i.e.

$$
\mu_{z_{0}, c}=\varepsilon_{z_{0}}^{\mathbf{c} \Omega}
$$

$\left.{ }^{2}\right)$ It is an easy and well known consequence of the Hopf maximum principle that $\Delta v-\partial v / \partial t<0$ for a $C^{2,1}$-function in a domain $D \subset \boldsymbol{R}^{p+1}$ implies that $v$ is supercaloric on $D$. 
The proof will be given in the next paragraph. It will lead also to the following addendum:

2.2' Addendum. In every heat ball $\Omega=\Omega\left(z_{0}, c\right)$ there exists a sequence $\left(z_{k}\right)$ of points in $\Omega$ such that the corresponding sequence $\left(\mu_{z_{k}}^{\Omega}\right)$ of harmonic measures converges vaguely to the Fulks measure $\mu_{z_{0}, c}$.

Let us remark that this Addendum can also be deduced from our Main Theorem and a lemma of Köhn and Sieveking [13] (see also [16], p. 356).

The Addendum $2.2^{\prime}$ leads directly to the mean value property (F) of Fulks:

2.3 Corollary 1. Let $h$ be a function which is caloric in an open set $U \subset \boldsymbol{R}^{p+1}$. Then the equality

$$
\int h d \mu_{z_{0}, c}=h\left(z_{0}\right)
$$

holds for all heat balls $\Omega\left(z_{0}, c\right)$ for which $\bar{\Omega}\left(z_{0}, c\right) \subset U$.

Proof. Suppose that $\left(z_{k}\right)$ is a sequence in $\Omega=\Omega\left(z_{0}, c\right)$ with the properties stated in the Addendum. Then

$$
\int h d \mu_{z_{k}}^{\Omega}=h\left(z_{k}\right) \text { for all } k \in \boldsymbol{N} .
$$

From this the result follows for $k \rightarrow+\infty$ since $h$ is continuous on $U$.

An immediate consequence of the Main Theorem and Proposition 2.1 is

2.4 Corollary 2. $z_{0}$ is the only irregular boundary point of $\Omega\left(z_{0}, c\right)$.

This result can be proved in several other ways. E.g. it follows from Proposition 2.1 and Watson [18], Theorem 9, p. 399. Another proof is indicated in the Remark following our Proposition 3.1.

We shall proeced now to more important consequences of the Main Theorem. As pointed out before, we consider $\boldsymbol{R}^{p+1}$ as a harmonic space with respect to the sheaf of caloric functions. The hyperharmonic (respectively superharmonic) functions for this harmonic space will be called hypercaloric (respectively supercaloric) functions. Consequently (see [1], p. 127), a hypercaloric function $u$ on an open set $U \subset \boldsymbol{R}^{p+1}$ satisfies the inequality

$$
\int u d \mu_{z}^{V} \leqq u(z)
$$

for every point $z \in U$ and every relatively compact, open neighborhood $V \subset \bar{V} \subset U$ of $z$. Here $\mu_{z}^{V}=\varepsilon_{z}^{c V}$ denotes again the harmonic measure. In particular, the approach of Gehring [11] - if extended to lower semicontinuous functions with values in ]$-\infty,+\infty]-$ leads to an equivalent definition of hypercaloric functions. This is an immediate consequence of Köhn [12], p. 5 (Satz 3).

In contrast to the class of hypercaloric functions there is an analogous class of functions introduced by Watson [18] in his thesis and used in subsequent investigations, in particular in [19]. The definition makes essential use of the Fulks measures: 
A function $u: U \rightarrow]-\infty,+\infty$ ] defined on an open subset $U$ of $\boldsymbol{R}^{p+1}$ is called a hypertemperature on $U$ if, besides being lower semicontinuous, it satisfies the inequality

$$
\int u d \mu_{z_{0}, c} \leqq u\left(z_{0}\right)
$$

for all choices of points $z_{0} \in U$ and real numbers $c>0$ for which the heat ball $\Omega\left(z_{0}, c\right)$ is contained together with its boundary $B\left(z_{0}, c\right)$ in $U$. We claim

2.5 Theorem. An extended real-valued function $u$ defined on an open subset $U$ of $\boldsymbol{R}^{p+1}$ is a hypertemperature on $U$ if and only if it is hypercaloric on $U$.

Proof. Suppose that $u$ is a hypertemperature on $U$ and that $V$ is a regular set such that $V \subset \bar{V} \subset U$. It suffices to prove that for every function $f \in \mathscr{C}(\partial V)$, i.e. continuous real-valued function on the boundary $\partial V$ of $V$, satisfying $f(z) \leqq u(z)$ for $z \in \partial V$ one has

$$
\int f d \mu_{z}^{V} \leqq u(z) \text { for all } z \in V .
$$

But since $z \mapsto H_{f}^{V}(z)=\int f d \mu_{z}^{V}$ solves the Dirichlet problem for the boundary function $f$ (according to the definition of a regular set), $H_{f}^{V}$ is a temperature on $V$ and, consequently, the function

$$
v(z)=u(z)-H_{f}^{V}(z) \quad(z \in V)
$$

is a hypertemperature on $V$. For such functions the boundary minimum principle is valid (see [18], p. $397^{3}$ )): So $v \geqq 0$ follows from

$$
\liminf _{\substack{z^{\prime} \rightarrow z \\ z^{\prime} \in V}} v\left(z^{\prime}\right)=\liminf _{\substack{z^{\prime} \rightarrow z \\ z^{\prime} \in V}} u\left(z^{\prime}\right)-\lim _{\substack{z^{\prime} \rightarrow z \\ z^{\prime} \in V}} H_{f}^{V}\left(z^{\prime}\right) \geqq u(z)-f(z) \geqq 0
$$

$(z \in \partial V)$. This proves (2.4). - Conversely, assume that $u$ is hypercaloric on $U$ and that $\bar{\Omega}\left(z_{0}, c\right) \subset U$ for some $z_{0} \in U$ and $c>0$. Since the closure $\bar{\Omega}$ of $\Omega=\Omega\left(z_{0}, c\right)$ is compact, $u$ is bounded from below on $\bar{\Omega}: u(z)>\gamma$ for all $z \in \bar{\Omega}$ and some $\gamma \in \boldsymbol{R}$. Consequently, there exists a relatively compact open set $U_{0}$ such that

$$
\bar{\Omega} \subset U_{0} \subset \bar{U}_{0} \subset U
$$

and

$$
u(z)>\gamma \text { for all } z \in U_{0} .
$$

Let us assume for a moment that $u$ is even bounded on $U_{0}$. Then, according to the extension theorem of [1], p. 159, there exists for a given open set $V$ satisfying $\bar{\Omega} \subset V \subset \bar{V} \subset U_{0}$ a potential $p \in \mathscr{C}\left(\boldsymbol{R}^{p+1}\right)$ which is even caloric on $V$ as well as a potential $\tilde{u}$ on $\boldsymbol{R}^{p+1}$ such that

$$
\tilde{u}(z)=u(z)+p(z) \text { for all } z \in V .
$$

${ }^{3}$ ) In order to be in formal agreement with [18], Theorem 8, it suffices to replace $u$ on $V$ by $\inf (u, \alpha)$ where $\alpha \in \boldsymbol{R}$ is an upper bound for $f$. 
It follows from our Main Theorem that, whenever $w$ is a hypercaloric function $\geqq 0$ on $\boldsymbol{R}^{p+1}$, we have

$$
\int w d \mu_{z_{0}, c} \leqq w\left(z_{0}\right) .
$$

(In fact, $\hat{R}_{w}^{E} \leqq w$ on $\boldsymbol{R}^{p+1}$ and

$$
\hat{R}_{w}^{E}\left(z_{0}\right)=\int w d \varepsilon_{z_{0}}^{E}
$$

according to the definition of the balayée $\hat{R}_{w}^{E}$ and the swept-out measure $\varepsilon_{z_{0}}^{E}$ for an arbitrary set $E \subset \boldsymbol{R}^{p+1}$, in particular for $E=\mathbf{C} \Omega$.) The inequality (2.8) can be applied to $\tilde{u}$ which yields

$$
\int \tilde{u} d \mu_{z_{0}, c} \leqq \tilde{u}\left(z_{0}\right) .
$$

Since $p$ is caloric on $V$ we also have

$$
\int p d \mu_{z_{0}, c}=p\left(z_{0}\right)
$$

according to Corollary 2.3. Since the Fulks measure lives on $B\left(z_{0}, c\right) \subset V$, it then follows from (2.7) and (2.8') that

$$
\int \tilde{u} d \mu_{z_{0}, c}=\int u d \mu_{z_{0}, c}+p\left(z_{0}\right) \leqq \tilde{u}\left(z_{0}\right) .
$$

Consequently, we have

$$
\int u d \mu_{z_{0}, c} \leqq u\left(z_{0}\right) .
$$

If $u$ is not bounded from above, we pass from $u$ to the function $u_{n}=\inf (u, n)$ which is hypercaloric for every $n \in N$. We then have proved

$$
\int u_{n} d \mu_{z_{0}, c} \leqq u_{n}\left(z_{0}\right)
$$

for all $n \in N$ and hence, by passing to the limit for $n \rightarrow+\infty$,

$$
\int u d \mu_{z_{0}, c} \leqq u\left(z_{0}\right) \text {. }
$$

This, however, proves that $u$ is a hypertemperature on $U$.

2.6 Remarks. 1) An immediate consequence of Theorem 2.5 is the result that the supercaloric functions on an open set $U$ coincide with the supertemperatures in the sense of Watson [18]. This follows immediately from [18], Lemma 5, by observing that finiteness of a function on a dense subset of an open set in $\boldsymbol{R}^{p+1}$ implies condition $\left(\delta_{3}\right)$ of [18].

2) Another consequence of Theorem 2.5 are the results of Fulks [10] mentioned at the end of our first paragraph: A function $h$ defined on an open set $U \subset \boldsymbol{R}^{p+1}$ is caloric if and only if the mean value property (1.13) holds for all heat balls $\Omega\left(z_{0}, c\right)$ for which $\bar{\Omega}\left(z_{0}, c\right) \subset U$.

We close this paragraph with a probabilistic interprelation of the Fulks measures. For this purpose we denote by $\left(X_{t}\right)_{t \geqq 0}$ or, more precisely by $\left(\Omega, \mathscr{F},\left(P^{z}\right)_{z \in R^{p+1}}\right.$, $\left.\left(X_{t}\right)_{t \geqq 0}\right)$ the well-known diffusion process with $\boldsymbol{R}^{p+1}$ as state space which is associ- 
ated to the heat equation (see Meyer [17], pp. 63-64). Hence $\left(X_{i}\right)_{t \geqq 0}$ is projected on the $p$-dimensional Brownian motion by the first canonical projection of $\boldsymbol{R}^{p+1}=$ $\boldsymbol{R}^{p} \times \boldsymbol{R}$ and on the deterministic movement with constant velocity -1 by the second canonical projection. We will call $\left(X_{t}\right)_{t \geqq_{0}}$ the Brownian heat transfer.

2.7 Proposition. For every heat ball $\Omega=\Omega\left(z_{0}, c\right)$ the Fulks measure $\mu_{z_{0}}, c$ is the distribution of the first hit of the complement of $\Omega$ by the Brownian heat transfer starting at $z_{0}$. More precisely,

$$
\mu_{z_{0}, c}=P^{z_{0}}\left(X_{T_{\mathbf{C} \Omega}}^{-1}\right)
$$

where $T_{\mathrm{C} \Omega}$ denotes the first hitting time of the complement of $\Omega$.

Proof. It is well known that the excessive functions of the Brownian heat transfer (or, more precisely, of its transition semigroup) coincide with the hypercaloric functions $\geqq 0$ defined on $\boldsymbol{R}^{p+1}$. (A proof of this fact can be found in Bliedtner [5], p. 96, where the situation is even more general.) The arguments in the proof of [2], Theorem 7.1, can then be repeated. As a result one obtains the identification of the balayée $\hat{R}_{u}^{A}$ with the function

$$
P_{A} u(z)=E^{z}\left(1_{\left\{T_{A}<+\infty\right\}} u \circ X_{T A}\right) \quad\left(z \in \boldsymbol{R}^{p+1}\right)
$$

for all hypercaloric functions $u \geqq 0$ on $\boldsymbol{R}^{p+1}$ and all Borel sets $A \subset \boldsymbol{R}^{p+1}$. For $A=\mathbf{C} \Omega$ the result then follows since the kernel $P_{\mathrm{C} \Omega} u(z)$ is just the integral of $u$ with respect to the hitting distribution $\tau=P^{z_{0}}\left(X_{T_{C \Omega}}^{-1}\right)$ appearing in the Proposition $\left.{ }^{4}\right)$. So we have, according to our Main Theorem,

$$
\int u d \mu_{z_{0}, c}=\hat{R}_{u}^{\mathbf{C} \Omega}\left(z_{0}\right)=\int u d \tau
$$

for all hypercaloric functions $u \geqq 0$ on $\boldsymbol{R}^{p+1}$. This implies $\tau=\mu_{z_{0}, c}$ (see [1], p. 113) and proves our result.

\section{Proof of the Main Theorem}

Let us put $\Omega=\Omega\left(z_{0}, c\right), B=B\left(z_{0}, c\right), q=q_{z_{0}}$ and

$$
\mu_{0}=\mu_{z_{0}, c} \quad\left(z_{0}=\left(x_{0}, t_{0}\right), c>0\right) \text {. }
$$

For $\left.c^{\prime} \in\right] 0, c$ [ the domains $\Omega\left(z_{0}, c^{\prime}\right)$ increase with $c^{\prime}$ and have $\Omega$ as their union:

$$
\bigcup_{0<c^{\prime}<c} \Omega\left(z_{0}, c^{\prime}\right)=\Omega\left(z_{0}, c\right) .
$$

Consequently, for a given point $(x, t) \in \Omega$ the number $\left.c^{\prime} \in\right] 0, c[$ can be chosen in such a way that $(x, t)$ lies in $\Omega\left(z_{0}, c^{\prime}\right)$; it suffices to choose

$$
t_{0}-t<c^{\prime}<c \text {. }
$$

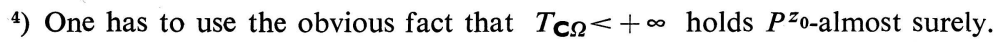


Since $c^{\prime}$ will be fixed for a while, we put $\Omega^{\prime}=\Omega\left(z_{0}, c^{\prime}\right)$ and $B^{\prime}=B\left(z_{0}, c^{\prime}\right)$. We then have

$$
\bar{\Omega}^{\prime} \backslash\left\{z_{0}\right\} \subset \Omega .
$$

Finally we introduce for an arbitrary set $A \subset \boldsymbol{R}^{p+1}$

$$
A_{t}=A \cap\left(\boldsymbol{R}^{p} \times\right]-\infty, t[) .
$$

We intend to study the generalized solution $H_{f}=H_{f}^{\Omega}$ in $\Omega$ for a given boundary function $f \in \mathscr{C}(B)$. At the beginning we imitate the original proof of the property (F) in Fulks [10]. The domain $\Omega_{t}^{\prime}$ is contained with its boundary in $\Omega$ according to (3.3). The boundary splits into three disjoint sets:

$$
\partial \Omega_{t}^{\prime}=B_{t}^{\prime} \cup I^{\prime}(t) \cup I_{0}^{\prime}(t)
$$

where

$$
I^{\prime}(t)=\left\{(\xi, t):\left\|\xi-x_{0}\right\|<F_{c^{\prime}}\left(t_{0}-t\right)\right\}
$$

and

$$
I_{0}^{\prime}(t)=\left\{(\xi, t):\left\|\xi-x_{0}\right\|=F_{c^{\prime}}\left(t_{0}-t\right)\right\}
$$

(and where $F_{c^{\prime}}$ is defined in (1.7)). Since $u=H_{f}$ is a caloric function on $\Omega$ and hence caloric in a neighborhood of $\bar{\Omega}_{t}^{\prime}$, an application of the Gauss divergence theorem ${ }^{5}$ ) yields as in [10]:

$$
\int_{M^{\prime}(t)}\left[\sum_{i=1}^{p}\left(u v_{\xi_{i}}-v u_{\xi_{i}}\right) n_{i}+u v n_{p+1}\right] d \sigma^{\prime}=0 .
$$

Here $\sigma^{\prime}$ is the surface measure of the smooth manifold

$$
M^{\prime}(t)=\partial \Omega_{t}^{\prime} \backslash I_{0}^{\prime}(t)
$$

and $n_{1}, \ldots, n_{p+1}$ are the components of the outer normal field. Furthermore, $v$ is a solution of the adjoint heat equation in an open neighborhood of $\bar{\Omega}_{t}^{\prime}$. We apply this formula (as in [10]) to two special functions $v$ : to the constant function 1 and to the function $k=W_{z_{0}}$ of (1.4). We then obtain

$$
\int_{M^{\prime}(t)}\left[-\sum_{i=1}^{p} u_{\xi_{i}} n_{i}+u n_{p+1}\right] d \sigma^{\prime}=0
$$

and

$$
\int_{M^{\prime}(t)}\left[\sum_{i=1}^{p}\left(u k_{\xi_{i}}-k u_{\xi_{i}}\right) n_{i}+u k n_{p+1}\right] d \sigma^{\prime}=0
$$

respectively. We also observe that

$$
\begin{gathered}
n_{1}=\ldots=n_{p}=0, \quad n_{p+1}=1 \text { on } I^{\prime}(t), \\
k=d^{\prime} \text { on } B^{\prime}
\end{gathered}
$$

$\left.{ }^{5}\right)$ For an appropriate form of this theorem including "singular" boundary points (in the sense of differential geometry) like those in $I_{0}^{\prime}(t)$ see König [14]. 
and

$$
-\sum_{i=1}^{p} k_{\xi_{i}} n_{i}=d^{\prime} q \text { on } \quad B^{\prime} \backslash\left\{z_{0}\right\}
$$

where

$$
d^{\prime}=\left(\frac{1}{4 \pi c^{\prime}}\right)^{p / 2}
$$

We then obtain, in view of (3.5),

$$
\int_{I^{\prime}(t)} u d \lambda^{p}=\int_{B_{t}^{\prime}}\left[\sum_{i=1}^{p} u_{\xi_{i}} n_{i}-u n_{p+1}\right] d \sigma^{\prime}
$$

and

$$
\int_{I^{\prime}(t)} u k d \lambda^{p}=d^{\prime} \int_{B_{t}^{\prime}}\left[\sum_{i=1}^{p} u_{\xi_{i}} n_{i}-u n_{p+1}\right] d \sigma^{\prime}+d^{\prime} \int_{B_{t}^{\prime}} u q d \sigma^{\prime},
$$

where $\lambda^{p}$ is the Lebesgue measure on $\boldsymbol{R}^{p} \times\{t\}$ (so that integration with respect to $\lambda^{p}$ refers to the space variable). From these two equalities we finally deduce

$$
\int_{I^{\prime}(t)} u\left(k-d^{\prime}\right) d \lambda^{p}=d^{\prime} \int_{B_{t}^{\prime}} u q d \sigma^{\prime} .
$$

Obviously, the restriction of $u=H_{f}$ to $\Omega_{t}$ extends to $\bar{\Omega}_{t}$ continuously by means of $f$. This follows from Proposition 2.1 since $z_{0} \notin \bar{\Omega}_{t}$. An elementary discussion of the surface measures $\sigma^{\prime}$ for $c^{\prime} \rightarrow c$ shows that we may pass to the limit for $c^{\prime} \rightarrow c$. We then obtain from (3.16)

$$
\int_{I(t)} H_{f} k d \lambda^{p}=d \int_{I(t)} H_{f} d \lambda^{p}+d \int_{B_{t}} f q d \sigma,
$$

where $I(t)$ is defined in the same way as $I^{\prime}(t)$ by replacing $c^{\prime}$ by $c$ in (3.16), where

$$
d=\left(\frac{1}{4 \pi c}\right)^{p / 2}
$$

and where $\sigma$ denotes the surface measure of $B$.

Next we study the behaviour of (3.17) for $t<t_{0}$ tending to $t_{0}$. We observe that

$$
\left|\int_{I(t)} H_{f} d \lambda^{p}\right| \leqq\|f\| \lambda^{p}(I(t))
$$

since $\left|H_{f}\right| \leqq\|f\|$ on $\Omega$, where $\|f\|$ denotes the sup-norm on $\mathscr{C}(B)$. We then obtain

$$
\lim _{t \rightarrow t_{0}-0} \int_{I(t)} H_{f} d \lambda^{p}=0
$$

since $I(t)$ is an open euclidean ball in $\boldsymbol{R}^{p} \times\{t\}$ with radius $F_{c}\left(t_{0}-t\right)$. The second term on the right-hand side of (3.17) has

$$
d \int_{B} f q d \sigma=\mu_{0}(f)
$$

as a limit for $t \rightarrow t_{0}-0$. So we know that the limit of the left-hand side of (3.17) exists, namely

$$
\lim _{t \rightarrow t_{0}-0} \int_{I(t)} H_{f} k d \lambda^{p}=\mu_{0}(f)
$$


By changing the space variable $\xi$ in the integral over $I(t)$ by means of

we obtain

$$
\eta=\frac{1}{\sqrt{4\left(t_{0}-t\right)}}\left(\xi-x_{0}\right)
$$

$$
\int_{I(t)} H_{f} k d \lambda^{p}=\pi^{-p / 2} \int_{K(t)} H_{f}\left(x_{0}+\sqrt{4\left(t_{0}-t\right)} \eta, t\right) e^{-\|\eta\|^{2}} d \eta
$$

where $K(t)$ is the open ball in $\boldsymbol{R}^{p}$ with the origin as center and with radius

$$
r(t)=\frac{F_{c}\left(t_{0}-t\right)}{\sqrt{4\left(t_{0}-t\right)}} .
$$

Since

we have

$$
\lim _{t \rightarrow t_{0}-0} r(t)=+\infty
$$

$$
\lim _{t \rightarrow t_{0}-0} \pi^{-p / 2} \int_{K(t)} e^{-\|\eta\|^{2}} d \eta=1
$$

By applying a mean value argument ${ }^{6}$ ) to the integral (3.20), we obtain for each $t$ satisfying $0<t_{0}-t<c$ the existence of a point $\eta_{t} \in K(t)$, hence of a point

$$
\left(\xi_{t}, t\right)=\left(x_{0}+\sqrt{4\left(t_{0}-t\right)} \eta_{t}, t\right) \in I(t)
$$

such that

$$
\int_{I(t)} H_{f} k d \lambda^{p}=\pi^{-p / 2} H_{f}\left(\xi_{t}, t\right) \int_{K(t)} e^{-\|\eta\|^{2}} d \eta .
$$

Consequently, it follows from (3.19)-(3.21) that

$$
\lim _{t \rightarrow t_{0}-0} H_{f}\left(\xi_{t}, t\right)=\mu_{0}(f) \text {. }
$$

Hereby, the point $\left(\xi_{t}, t\right) \in I(t)$ depends on the given boundary function $f \in \mathscr{C}(B)$.

So we have proved the following result:

3.1 Proposition. For every $f \in \mathscr{C}(B)$ and every $t \in] t_{0}-c, t_{0}[$ there exists $a$ point $\xi_{t}$ - depending on $f$ - in the p-dimensional ball

such that

$$
\left\|\xi-x_{0}\right\|<F_{c}\left(t_{0}-t\right)
$$

$$
\lim _{t \rightarrow t_{0}-0} H_{f}\left(\xi_{t}, t\right)=\mu_{0}(f) .
$$

3.2 Remark. Corollary 2.4 follows already from this Proposition. In fact, $z_{0}$ cannot be regular since otherwise (3.22) would imply $f\left(z_{0}\right)=\mu_{0}(f)$ for all $f \in \mathscr{C}(B)$,

$\left.{ }^{6}\right)$ We are referring to the following fact which can te proved easily: Let $\mu$ be a positive bounded Radon measure on a connected, locally compact space $Y$ with support equal to $Y$. Then for every bounded function $g \in \mathscr{C}(Y)$ there exists a point $\eta \in Y$ such that

$$
\int g d \mu=g(\eta)\|\mu\| \text {. }
$$


and hence $\mu_{0}=\varepsilon_{z_{0}}$. However, $\mu_{0}=\mu_{z_{0}, c}$ has a density with respect to the surface measure $\sigma$ which implies $\mu_{0}\left(\left\{z_{0}\right\}\right)=0$.

We continue with the proof of the Main Theorem and claim:

3.3 Lemma. Let $\Lambda$ denote the set of all measures

$$
\lambda=\alpha \varepsilon_{z_{0}}+(1-\alpha) \varepsilon_{z_{0}}^{c \Omega}, \quad 0 \leqq \alpha \leqq 1 .
$$

Then for every function $f \in \mathscr{C}(B)$ there exists a measure $\lambda \in \Lambda$ such that $\lambda(f)=\mu_{0}(f)$.

Proof. According to Proposition 3.1, for every $f \in \mathscr{C}(B)$ there exists a sequence $\left(z_{n}\right)$ in $\Omega$ converging to $z_{0}$ such that

$$
\lim _{n \rightarrow \infty} \mu_{z_{n}}^{\Omega}(f)=\mu_{0}(f)
$$

holds for the corresponding sequence of harmonic measures. From this sequence (of probability measures) we may extract a subsequence $\left(\mu_{z_{n_{k}}}^{\Omega}\right)$ which converges vaguely to a probability (Radon) measure $\lambda$ on $B$ depending on $f$ (see [4], p. 243). So (3.23) implies $\lambda(f)=\mu_{0}(f)$. We can now apply - and this is crucial - a result of Boboc and Cornea [6] (for a simple proof see Lukeš and Malý [16], p. 362). According to it, the measure $\lambda$ is of the form

$$
\lambda=\alpha \varepsilon_{z_{0}}+(1-\alpha) \varepsilon_{z_{0}}^{\mathbf{C} \Omega \backslash\left\{z_{0}\right\}} \quad(0 \leqq \alpha \leqq 1) .
$$

But since all points in $\boldsymbol{R}^{p+1}$ are polar for the underlying harmonic structure (see [1], p. 79), we have

$$
\varepsilon_{z_{0}}^{\mathrm{C} \Omega \backslash\left\{z_{0}\right\}}=\varepsilon_{z_{0}}^{C \Omega}
$$

(see [1], pp. 83 and 99). This proves $\lambda \in \Lambda$, and hence the Lemma.

The remaining part of the proof of the Main Theorem is now pure linear algebra. It suffices to apply the next lemma to the case $X=B, x_{0}=z_{0}, v=\varepsilon_{z_{0}}^{\mathrm{c} \Omega}$. In fact, it follows from Lemmas 3.3 and 3.4 that $\mu_{0}$ is in $\Lambda$ which means

$$
\mu_{0}=\alpha \varepsilon_{z_{0}}+(1-\alpha) \varepsilon_{z_{0}}^{\mathbf{c} \Omega} \text { for some } \alpha \in[0,1] .
$$

Since $\mu_{0}$ has a density with respect to $\sigma$, we have

$$
0=\mu_{0}\left(\left\{z_{0}\right\}\right)=\alpha+(1-\alpha) \varepsilon_{z_{0}}^{\mathbf{c} \Omega}\left(\left\{z_{0}\right\}\right) \geqq \alpha
$$

which implies $\alpha=0$ and hence $\mu_{0}=\varepsilon_{z_{0}}^{\mathbf{c} \Omega}$, the statement of the Main Theorem.

3.4 Lemma. Let $X$ be a compact space, $x_{0} \in X$ and $v$ a positive Radon measure on $X$. Suppose that a Radon measure $\mu_{0}$ on $X$ has the property that for every $f \in \mathscr{C}(X)$ there exists a number $\alpha_{f} \in[0,1]$ such that $\mu_{0}(f)=\lambda(f)$, where $\lambda=\alpha_{f} \varepsilon_{x_{0}}+$ $\left(1-\alpha_{f}\right) v$. Then $\mu_{0}$ is of the form $\mu_{0}=\alpha \varepsilon_{x_{0}}+(1-\alpha) v$ for some $\alpha \in[0,1]$.

Proof. Let us denote by $\Lambda_{v}$ the set of all measures $\lambda=\alpha \varepsilon_{x_{0}}+(1-\alpha) v$ with $\alpha \in[0,1]$ and by $H$ the set of all functions $h \in \mathscr{C}(X)$ satisfying $v(h)=h\left(x_{0}\right)$. Then 
obviously, for all $\lambda \in \Lambda_{v}$ and $h \in H$,

$$
\lambda(h)=v(h)=h\left(x_{0}\right) .
$$

Our condition therefore implies

$$
\mu_{0}(h)=\lambda(h) \text { for all } \lambda \in \Lambda_{v} \text { and } h \in H .
$$

Consequently, in the case $H=\mathscr{C}(X)$, i.e. $v=\varepsilon_{x_{0}}$, the measure $\mu_{0}$ equals $\varepsilon_{x_{0}}$ and thus is of the desired form. In the remaining case where $H \neq \mathscr{C}(X)$ we choose $g \in$ $\mathscr{C}(X) \backslash H$. The corresponding measure $\lambda_{0}=\alpha_{g} \varepsilon_{x_{0}}+\left(1-\alpha_{g}\right) v \in \Lambda_{v}$ satisfying $\lambda_{0}(g)=$ $\mu_{0}(g)$ is then uniquely determined: the coefficient $\alpha_{g}$ equals

$$
\alpha_{g}=\frac{\mu_{0}(g)-v(g)}{g\left(x_{0}\right)-v(g)} .
$$

Since $H$ is a hyperplane in $\mathscr{C}(X)$ - defined by the linear form $v-\varepsilon_{x_{0}} \neq 0$ - every $f \in \mathscr{C}(X)$ is of the form $f=h+\tau g$ with some $h \in H$ and $\tau \in \boldsymbol{R}$. For the measure $\lambda_{0}$ we then have

$$
\mu_{0}(g)=\lambda_{0}(g) \text { and } \mu_{0}(h)=\lambda_{0}(h)
$$

the latter according to (3.24), and, consequently,

$$
\mu_{0}(f)=\mu_{0}(h)+\tau \mu_{0}(g)=\lambda_{0}(h)+\tau \lambda_{0}(g)=\lambda_{0}(f) .
$$

Since $\lambda_{0}$ does not depend on the choice of $f$, this proves $\mu_{0}=\lambda_{0}$. Hence, also in this case, $\mu_{0}$ has the desired form.

As a corollary, we now obtain also a proof of the Addendum 2.2': For every $f \in \mathscr{C}(B)$, the proof of Lemma 3.3 shows the existence of a sequence $\left(z_{k}\right)$ in $\Omega$ converging to $z_{0}$ such that the corresponding sequence $\left(\mu_{z_{k}}^{\Omega}\right)$ of harmonic measures converges to a measure $\lambda \in \Lambda$ which satisfies $\lambda(f)=\mu_{0}(f)$. Since $\mu_{0} \neq \varepsilon_{z_{0}}$, the function $f \in \mathscr{C}(B)$ may be chosen in such a way that $\mu_{0}(f) \neq f\left(z_{0}\right)$. We have $\lambda=$ $\alpha \varepsilon_{z_{0}}+(1-\alpha) \mu_{0}$ for some $\alpha \in[0,1]$ according to the Main Theorem. Consequently, $\mu_{0}(f)=\alpha f\left(z_{0}\right)+(1-\alpha) \mu_{0}(f)$ which implies $\alpha=0$ and thus $\lambda=\mu_{0}$.

3.5 Remark. The set $\Lambda$ of Lemma 3.3 coincides with the set of all cluster points of $\left(\mu_{z}^{\Omega}\right)$ for $z \rightarrow z_{0}, z \in \Omega$. This is an easy consequence of Lukeš and Malý [16], Theorem 16. In fact, we know from Corollary 2.4 that all boundary points of $\Omega$ are regular, wi.h $z_{0}$ as the only exception. From this the above result follows from [16], Theorem 16, in the same way as Corollary 17 of [16] was derived from that Theorem.

\section{References}

[1] BAUER, H.: Harmonische Räume und ihre Potentialtheorie. - Lecture Notes in Mathematics

22. Springer-Verlag, Berlin-Heidelberg-New York, 1966.

[2] BAUER, H.: Harmonic spaces and associated Markov processes. - Potential Theory, C.I.M.E.,

I Ciclo, Stresa, 1969. Edizioni Cremonese, Roma, 1970, 23-67. 
[3] BAUER, H.: Harmonische Räume. - Jahrbuch Überblicke Mathematik 1981. Bibliographisches Institut, Mannheim-Wien-Zürich, 1981, 9-35.

[4] Bauer, H.: Probability theory and elements of measure theory, 2nd edition. - Academic Press. Inc., London-New York-Toronto-Sydney-San Francisco, 1981.

[5] Bliedtner, J.: Harmonische Gruppen und Huntsche Faltungskerne. - Seminar über Potentialtheorie (Edited by H. Bauer). Lecture Notes in Mathematics 69. Springer-Verlag, Berlin-Heidelberg-New York, 1968, 69-102.

[6] Вовос, N., et A. Cornea: Comportement des balayées des mesures ponctuelles. Comportement des solutions du problème de Dirichlet aux points irréguliers. - C. R. Acad. Sci. Paris Sér. A 264, 1967, 995-997.

[7] Constantinescu, C., and A. Cornea: Potential theory on harmonic spaces. - Die Grundlehren der mathematischen Wissenschaften 158. Springer-Verlag, Berlin-HeidelbergNew York, 1972.

[8] Effros, E. G., and J. L. Kazdan: On the Dirichlet problem for the heat equation. - Indiana Univ. Math. J. 20, 1971, 683-693.

[9] Friedman, A.: Partial differential equations of parabolic type. - Prentice Hall, Inc., Englewood Cliffs, N. J., 1964.

[10] Fulks, W.: A mean value theorem for the heat equation. - Proc. Amer. Math. Soc. 17, 1966, $6-11$.

[11] Gehring, F. W.: On solutions of the equation of heat conduction. - Michigan Math. J. 5, 1958, 191-202.

[12] KöHN, J.: Harmonische Räume mit einer Basis semiregulärer Mengen. - Seminar über Potentialtheorie (Edited by H. Bauer). Lecture Notes in Mathematics 69. Springer-Verlag, Berlin-Heidelberg-New York, 1968, 1-12.

[13] KöHN, J., und M. Sieveking: Reguläre und extremale Randpunkte in der Potentialtheorie. Rev. Roumaine Math. Pures Appl. 12, 1967, 1489-1502.

[14] KöNIG, H.: Ein einfacher Beweis des Integralsatzes von Gauß. - Jber. Deutsch. Math.-Verein. 66, 1964, 119-138.

[15] Kuptsov, L. P.: Mean property for the heat-conduction equation. - Mat. Zametki 29, 1981, 211 223. [Russian; English translation: Math. Notes 29, 1981, 110-116.]

[16] Lukeš, J., and J. MALÝ: On the boundary behaviour of the Perron generalized solution. - Math. Ann. 257, 1981, 355-366.

[17] Meyer, P.-A.: Processus de Markov. - Lecture Notes in Mathematics 26. Springer-Verlag, Berlin-Heidelberg-New York, 1967.

[18] Watson, N. A.: A theory of subtemperatures in several variables. - Proc. London Math. Soc. (3) $26,1973,385-417$.

[19] Watson, N. A.: Green functions, potentials, and the Dirichlet problem for the heat equation. - Proc. London Math. Soc (3) 33, 1976, 251-298. Corrigendum: ibid. (3) 37, 1978, $32-34$.

Universität Erlangen--Nürnberg

Mathematisches Institut

Bismarckstraße $1 \frac{1}{2}$

D-8520 Erlangen

Federal Republic of Germany

Received 26 January 1984 